\title{
Das ciências sociais para as ciências sociais em saúde: a produção científica de pós-graduação em ciências sociais
}

\author{
Social sciences to social sciences in health: \\ the scientific production of graduation \\ programs in social sciences
}

Regina M aria Giffoni M arsiglia 1

Selma Patti Spinelli 1

$M$ arina França Lopes 1

Thiago Carvalho Pelucio Silva 1

\footnotetext{
1 Departamento de M edicina Social, Faculdade de Ciências M édicas da Santa Casa de São Paulo. Rua Dr. Cesário M ota Júnior 61 , 50 andar, Higienópolis, 01221-000, São Paulo SP. epidemio@santacasa.org.br
}

Abstract This article presents some features of the intellectual production developed into the disciplines of social and human sciences ending health, during the 90 's and the years of 2000 and 2001 in São Paulo. We initiated by identifying these works in the libraries of U niversidade de São Paulo, U niversidade Estadual de Campinas, Pontifícia U niversidade Católica de São Paulo and Escola de Administração de Empresas of Fundação Getúlio Vargas de São Paulo. The 258 mastering dissertations and doctoring thesis were found and classified according to: academic institution, year of conclusion, degree of postgraduation, disciplines, the matic approaching and advisers. Those teachers that oriented most of these works were interviewed with the purpose of knowing their experiences and thinking about the inclusion of health as an investigation matter in the programs of post graduation among the disciplines of sociology, anthropology, politics, geography, history, administration, law, economics, education, social psychology and social service.

Key words Postgraduation production, Social sciences, H uman sciences, $\mathrm{H}$ ealth
Resumo Este artigo apresenta al gumas características da produção intelectual desenvolvida em disciplinas de pós-graduação das ciências sociais e humanas ten do como objeto a saúde, durante os anos 90 e nos anos de 2000 e 2001 em São Paulo. Partimos da identificação desses trabalhos nas bibliotecas da U niversidade de São Paulo, U niversidade Estadual de Campinas, Pontifícia Universidade Católica de São Paulo e Escola de Administração de Empresas da Fundação G etúlio Vargas de São Paulo. As 258 dissertações de mestrado e teses de dou torado encontradas foram classificadas de acordo com: instituição de ensino, ano de conclusão, nível de pós-graduação, disciplinas, temáticas abordadas e orientadores. 0 s professores que orientaram o maior número desses trabalhos foram entrevistados com o intuito de conhecermos suas experiências e reflexões sobre a inserção da saúde como objeto de investigação nos programas de pós-graduação das disciplinas de antropologia, sociologia, política, geografia, história, administração, direito, economia, educação, psi cologia social e serviço social. Palavras-chave Produção de pós-graduação, Ciências sociais, Ciências humanas, Saúde 


\section{Explicações sociais sobre a saúde e a doença: notas históricas}

Percorrendo a história da medicina podemos perceber que desde a Antiguidade, seja em textos egípcios ou gregos, autores já apontavam relações entre as condições de vida e trabalho da população e a sua saúde. M ás condições de habitação ou de alimentação, excesso de trabalho ou más condições de trabalho, especialmente os riscos do trabal ho nas minas, aparecem em textos dos séculos 17 e 18. Os trabalhos se multiplicam e em 1700 o médico Bernardino Ramazzini apresentou seu tratado sobre as doenças dos artesãos (Sigerist, 1974). A partir do século 19 , e especialmente com o desenvolvimento da Revolução Industrial surge a medicina social, com investigações sistemáticas, na primeira metade do século 19, sobre as relações entre saúde e condições de vida: Virshow afirmava na primeira metade do século 19 que a medicina era social e que as epidemias ocorriam quando a população era "submetida a condições de vida diferentes" daquelas em que viviam normalmente, decorrentes de graves crises agrícolas, climáticas, guerras ou grau de exploração socioeconômica (Rosen, 1958). Villermé apresentou estudos apontando as taxas de mortalidade entre as diferentes classes sociais na França e entre os diferentes bairros de Paris (Rosen, 1980); Snow apontou as causas e os modos de transmissão do cólera em Londres, trabalho que é considerado por muitos o marco inicial da epidemiologia científica, é também um verdadeiro survey sociológico, em que se apontam as relações entre a epidemia e as condições socioeconômicas (Luz, 2000).

Ao final do século 19, no entanto, o desenvolvimento da bacteriologia, da farmacologia, e de um substrato científico para a medicina, afastou as explicações sociais dos estudos sobre a doença e a saúde, chegando-se praticamente a uma cisão entre as ciências humanas e sociais e as ciências da natureza, tanto do ponto de vista conceitual quanto do ponto de vista metodológico (Luz, 2000).

A partir do século 20, após a Primeira Guerra M undial, constitui-se o campo da saúde pública, e novamente as informações de caráter socioeconômico passaram a ser consideradas fatores de risco para a situação de saúde da coletividade; mas as ciências humanas e sociais como área de conhecimento permaneceram fora do campo da saúde até os anos 60 (Luz, 2000).
Para Nunes (1999), a preocupação da sociologia com a medicina, com a saúde e a doença volta a ser significativa no início dos anos 20 na sociologia norte-americana, na primeira Escola de Chicago e na U niversidade de Columbia, especialmente os trabal hos de Sigerist (1929; 1946) eStern (1940); H all (1948). A pós a Segunda Guerra, afirma Nunes (1999), a sociologia médica se transformou em atividade regular com apoio financeiro federal nos EUA, especialmente para os estudos que relacionavam classe social edoença mental. Foi criada uma teoria do comportamento na U niversidade de Chicago, articulando a sociologia, antropologia, psicologia, biologia comportamental, economia, ciência política e história. N os anos 60 expandiuse 0 interesse pela pós-graduação e a produção científica, e abrangeu uma diversidade de temas tais como uso de serviços pela população, comportamento diante da doença e da morte (Nunes, 1999).

No Brasil, desde a constituição das diversas disciplinas das ciências sociais a partir dos anos 40 elas também já passaram a ser incorporadas nos cursos de higiene e saúde pública, ena segunda metade dos anos 60 , passaram a fazer parte do ensino nos departamentos de medicina e diferentes cursos da área de saúde, sob a denominação de ciências da conduta ou ciências sociais aplicadas, com enfoque comportamental e culturalista, sob influência norte-americana (Canesqui, 1995).

No início dos anos 70, as ciências da conduta ou ciências sociais aplicadas foram criticadas como inadequadas para a explicação dos processos sociais, da situação da saúde e das políticas de saúde, temas que exigem abordagens que contemplem a análise da estrutura social e dinâmica das sociedades capital istas tardias ( Canesqui, 1995).

A partir de então, as análises macroestruturais, com apoio da sociologia e da ciência política passaram a predominar nos programas das disciplinas de ciências sociais incorporadas aos departamentos de medicina preventiva/social. Encontraram também nos cursos de pós-graduação e na constituição do novo campo de investigação denominado de "saúde coletiva" um substrato fértil para a produção teórica, eno interior da área, a definição de uma subárea, denominada de ciências sociais em saúde.

Podemos observar que durante a década de 1980, na subárea de ciências sociais em saúde cresceu o interesse por conceitos e autores das ciências políticas, acompanhando na práti- 
ca a proposição de um Sistema Único de Saúde - SUS, para o país, como suporte para o M ovimento da Reforma Sanitária que levou essa proposição à Constituição Federal de 1988. Partiuse de uma concepção ampla do processo saúde/ doença, enfatizando seus determinantes socioeconômicos, a partir das condições de vida e trabalho; da discussão sobre a necessidade de definição de um sistema de saúde baseado no conceito de cidadania; e da preocupação com 0 controle da sua implantação pela sociedade civil organizada.

Durante os anos 90, no entanto, percebe-se que $a$ antropologia e a psicologia social passaram a ter maior visibilidade na subárea de ciências sociais em saúde, ressaltando-se as preocupações com as representações sociais sobre o processo saúde/doença, a morte e o uso dos serviços de saúde por diferentes grupos sociais. Pode-se dizer que houve consolidação do ensino das ciências sociais nos cursos de pós-graduação em saúde pública e saúde coletiva e nos cursos de graduação em medicina, enfermagem e odontologia e implantação de alguns conteúdos ou disciplinas nos cursos de fisioterapia, fonoaudiologia e terapia ocupacional ( $M$ arsiglia $\&$ Spinelli, 1995).

Na década de 1990 houve também a criação de fóruns próprios para o debate das ciências sociais em saúde, a partir da comissão de ciências sociais criada durante os anos 80 no interior da Associação Brasileira de Pós-Graduação em Saúde Coletiva (Abrasco). Realizaram-se: 0 I Encontro Nacional de Ciências Sociais em Saúde (1993; Belo Horizonte), a Oficina de Ciências Sociais em Saúde (1995; Rio de Janeiro), o 1o Congresso Brasileiro de Ciências Sociais em Saúde (1995; Curitiba), o 2o Congresso Brasileiro de Ciências Sociais em Saúde (1995; São Paulo). Houve, por fim, em dezembro de 2002, um seminário cujo objetivo foi contextualizar, do ponto de vista da sociologia, da antropologia e da ciência política, o momento atual da saúde pública brasileira e o estado da arte do conhecimento em ciências sociais e saúde. Esse encontro foi organizado pela Comissão de Ciências Sociais da Abrasco em parceria com a Casa de O swal do Cruz e o Departamento de Ciências Sociais da Escola N acional de Saúde Pública, no Rio de Janeiro.

\section{Ampliação e aprofundamento das ciências sociais e humanas no campo da saúde nos anos 90}

Questão importante a assinal ar é que durante os anos 90 foi se ampliando o interesse do campo da saúde para as ciências humanas em geral, o que pode ser demonstrado pela produção na área de saúde coletiva e nos encontros e congressos próprios das ciências sociais em saúde.

A formação de cientistas sociais para a área da saúde é uma preocupação constante nos vários fóruns das ciências sociais em saúde, o que na prática vem sendo feito primordialmente pelos cursos de pós-graduação em saúde pública ou saúde coletiva. Tem-se a impressão de que não há grande interesse dos alunos dos cursos de graduação em ciências sociais pelas temáticas da saúde, muito embora o CN Pq reconheça uma subárea da sociologia da saúde. Alguns cursos de ciências sociais no início dos anos 90 desenvolveram discussões sobre as questões de saúde na graduação ou pós-graduação, mas os cientistas sociais da saúde, consideram que foram ações "eventuais" ou pelo menos "insuficientes" para criar uma alternativa de escolha profissional e acadêmica para os alunos de ciências sociais, como afirmam ( $M$ arsiglia $\&$ Spinelli, 1995).

N ossa intenção é apresentar o "o outro lado" da questão das ciências sociais em saúde, isto é, conhecer como a saúde foi tomada como objeto de pesquisa nos programas de pós-graduação em ciências sociais e humanas nesta última década. Para tanto coordenamos um levantamento nas bibliotecas de três universidades de São Paulo: Pontifícia Universidade Católica de São Paulo, Universidade de São Paulo, U niversidade Estadual de Campinas, e na Escola de Administração de Empresas da Fundação Getúlio Vargas de São Paulo. Tentamos também obter informações nas bibliotecas da U niversidade Estadual de São Paulo, mas em função da existência de diversos campi e do uso de programas diferentes nas bibliotecas, não nos foi possível obtêlas.

0 que temos a apresentar são resultados preliminares sobre a produção em saúde, nos cursos de ciências sociais e humanas dessas instituições de ensino de São Paulo, e assinalar tendências que deverão ser averiguadas em outras universidades e em outros estados do país.

As dissertações de mestrado e teses de doutorado foram classificadas por: instituição de ensino, ano de conclusão, nível de pós-gradua- 
ção, disciplinas, temáticas abordadas e orientadores. Os professores mais citados como orientadores foram entrevistados com o intuito de conhecer suas experiências nesse trabalho e reflexões acerca da inserção da saúde como objeto de estudo e pesquisa em sua área. Ao final obtivemos o depoimento de sete professores: dra. M aria H elena Oliva Augusto (Departamento de Sociologia/USP), dr. Pedro Luiz de Barros Silva (Instituto de Economia/U nicamp), dra. Ana M aria M alik (FGV/PROAHSA), dr. Luciano Prates Junqueira (pós-graduação em administração/PU C/SP), dra. M aria H elena Villas-Boas Concone (pós-graduação em ciências sociais/PU C/SP), dra. M ary Jane Paris Spink (pós-graduação em psicologia social/PUC/SP) e dra. Ivone Avelino (pós-graduação em história/ PUC/SP).

\section{Principais resultados}

Encontramos nas quatro instituições de ensino 258 dissertações de mestrado e teses de doutorado elaboradas entre 1990 e 2001 que abordaram temáticas da área de saúde. N os programas dePós-Graduação da PUC/SP e da USP encontramos o mai or número de trabalhos, respectivamente, 98 trabalhos (38\%) e 96 (36\%). Chama atenção, no entanto, a presença de $15 \%$ dos trabalhos na Fundação Getúlio Vargas, isto é, um número absoluto de 39 trabalhos, considerando-se que, ao contrário das demais instituições pesquisadas, ela conta apenas com um curso de graduação em administração de empresas, enquanto as demais têm vários cursos na área de ciências sociais e humanas. A criação do Programa de Administração H ospitalar de Sistemas de Saúde (PROAHSA) incentivou a produção intelectual da FGV/SP sobre questões da área da saúde, pois ao final da década de 1980 já haviam sido defendidas 20 dissertações de mestrado com temáticas de saúdee, dos anos 90 até 2001, contabilizamos os 39 trabalhos citados (Figura 1).

A distribuição dos trabalhos durante a década não é homogênea, havendo uma clara concentração de dissertações e teses concluídas entre 1995 e 1999 (48,9\%). 0 interesse pela saúde parece continuar grande nos cursos pesquisados, pois entre 2000 e 2001, encontramos 63 teses defendidas, isto é, $24,4 \%$ do total da produção levantada. Isoladamente, o maior número de teses/dissertações sobre a saúde concluídas nessas instituições foi encontrado nos anos de 1996 e 2001.

\section{Figura 1}

Dissertações e teses sobre saúde por universidades, 2002.

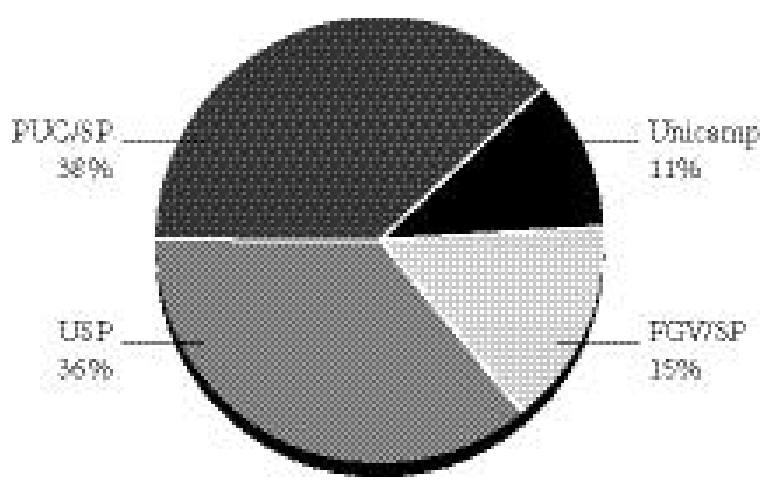

Fonte: Bibliotecas PUC/SP, Unicamp, USP e FGV/SP, 2002
Entre as universidades, se considerarmos os anos de maior produção, podemos verificar que na USP e na FGV/SP houve uma curva ascendente durante toda a década, um pouco mais acentuada entre 1998-1999. N a PUC/SP houve também um crescimento do número de trabaIhos em toda a década, mas com dois picos de produção em 1996 e 2001. Na U nicamp, houve um crescimento mais acentuado na primeira metade dos anos 90 , e diminuição do número de trabalhos a partir de 1995, mantendo-se em patamar menor até o final da década e início deste século.

A maioria dos trabalhos (quase $65 \%$ ) são dissertações de mestrado, mas se anal isarmos no interior de cada instituição de ensino há variações: na FGV/SP, mais de $90 \%$ foram dissertações de mestrado ena PU C/SP, perto de $80 \%$. Já na Unicamp ena USP há um certo equilíbrio entre a produção: praticamente metade dos trabalhos foi de mestrado e metade de doutorado.

Quanto aos cursos e programas de pós-graduação dos quais se originou essa produção intelectual, podemos apontar onze áreas diferentes: os mais freqüentes foram os da área de administração, com 49 trabalhos, isto é, 19\% do total, fazendo-se o reparo de que só na pós-graduação da FGV/SP foram concluídos 39 trabaIhos, isto é, quase $80 \%$ dos trabal hos da área de administração, enquanto a soma da produção de dissertações e teses sobre saúde nos programas de pós-graduação em administração da USP e PUC/SP atinge um total de 10 trabalhos.

Os cursos de pós-graduação em psicologia social foram os que produziram o segundo 
maior número de trabalhos sobre saúde, isto é, 47 teses (18,2\%) com especial destaque para a psicologia social da USP ea psicologia social da PUC/SP.

Em seguida estão os programas de pósgraduação em serviço social, que só existe na PUC/SP, com 30 trabalhos (11,6\%), os de educação com 29 trabal hos (11,2\%), com destaque especial para o da USP e, em seguida, para o da PUC/SP

As disciplinas clássicas das ciências sociais antropologia, política e sociologia - foram responsáveis, juntas, por 53 trabalhos, isto é, 20,5\%, e o maior número destes, foi produzido na sociologia da USP, que apresentou mais da metade desses trabalhos. Os programas de pós-graduação em antropologia, política, história, economia e direito apresentaram cada um em média de 13 a 16 trabalhos sobre temáticas de saúde, sendo que houve destaque para o número de trabalhos nas áreas de antropologia e direito na PUC/SP, na área de história da USP e de economia na Unicamp. Quanto aos trabalhos da área de política, estes estiveram distribuídos equilibradamente entre as três universidades. Os programas de pós-graduação dos cursos de geografia foram os que apresentaram o menor número de trabalhos, isto é, 8 (3,1\%) e praticamente, a maioria na USP. Diante disso fica claro queéinteressante incorporar-se as áreas de ciências humanas na área de saúde coletiva, além das disciplinas clássicas das ciências sociais (Figura 2).

As temáticas abordadas nos trabal hos são muito variadas e foram agrupadas de acordo com os mesmos critérios de classificação usados no interior da área de saúde coletiva, para podermos compará-las com o estudo apresentado por M inayo (2000).

Quando estão incluídos os trabal hos da pósgraduação da FGV/SP, as temáticas referentes às "políticas, instituições e gestão de serviços" foram 0 objeto de investigação em 31,4\% dos trabal hos. Em seguida ficaram as temáticas referentes aos "processos saúde/doença", abordadas em $27,9 \%$ dos trabalhos. Os temas relativos à "formação de recursos humanos" e os temas denominados "emergentes" apareceram respectivamente em $16,7 \%$ e $17,4 \%$ dos trabalhos. Os temas referentes aos "movimentos sociais" foram os menos abordados, como também constatou M inayo (2000), na pesquisa sobre a produção das ciências sociais em saúde no interior da saúde coletiva (Figura 3).

Se separarmos a produção da FGV/SP, há uma inversão, pois no conjunto da produção da
USP, PU C/SP e U nicamp, houve um maior interesse pelas temáticas de "processos saúde/doença", e em segundo lugar, pelas temáticas de "políticas, instituições e gestão de serviços". E se separarmos cada instituição acadêmica, veremos que na USP, pela ordem, houve preferência pelas temáticas de: "processos saúde/doença", "políticas, instituições e gestão de serviços", "temas emergentes", e um pequeno interesse pelos temas de "formação de recursos humanos" e "movimentos sociais". Na PU C/SP, um maior número de trabalhos em "formação de recursos humanos", seguido dos trabal hos sobre os "processos saúde/doença", e dos "temas emergentes", com menor número de trabalhos sobre "políticas, instituições e gestão de serviços". $\mathrm{Na}$ Unicamp os temas mais abordados foram os de "processos saúde/doença", "formação de recursos humanos", "temas emergentes" e poucos trabal hos sobre "movimentos sociais". No caso da Pós-Graduação da Escola de Administração de Empresas da FGV/SP, os temas predominantemente abordados foram os referentes às "políticas, instituições e serviços de saúde", seguidos dos temas referentes à "formação de recursos humanos".

Quando analisamos os temas das quatro instituições acadêmicas constatamos que, no nível do mestrado, foram elaborados quase $70 \%$ dos trabalhos sobre saúde, e o maior número foi sobre "políticas, instituições e gestão de serviços". Se separarmos a produção de pós-graduação da FGV/SP, podemos verificar que na USP, Unicamp e PUC/SP o maior número de dissertações de mestrado dedicou-se a temas referentes aos "processos saúde/doença". 0 mesmo ocorreu com os "temas emergentes", que apareceram em segundo lugar nas dissertações de mestrado do conjunto dos trabal hos da PU C/SP, USP eUnicamp. Ao contrário, ao juntarmos as dissertações da FGV/SP, a questão da "formação de recursos humanos" aparece em segundo lugar como tema abordado no número total de trabalhos.

$N$ as teses de doutorado, nível em que foram elaborados pouco mais de $30 \%$ dos trabalhos, os temas referentes às "políticas, instituições e gestão de serviços" foram os mais freqüentes, seguidos dos temas relativos aos "processos saúde/doença". Quanto aos "movimentos sociais", foram os temas menos abordados em todas as instituições acadêmicas estudadas, tanto em trabalhos de mestrado quanto de doutorado, como também encontrou M inayo (2000), ao estudar a produção no interior das ciências sociais em saúde. 
Figura 2

Dissertaç̃̃es e teses sobre saúde por cursos na FGV/SP, PU C/SP, U NICAM P e USP (1990-2001).

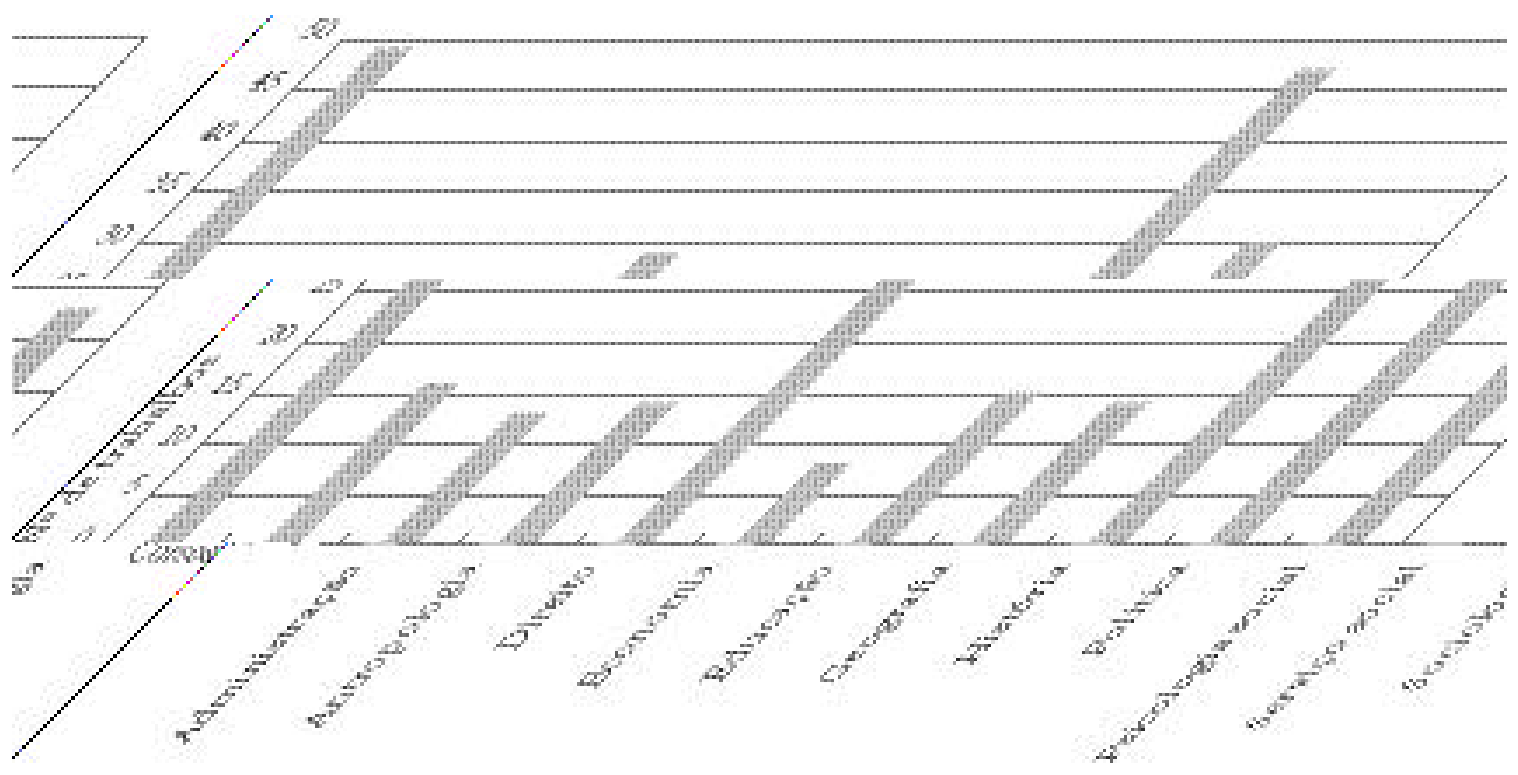

Fonte: Bibliotecas PUC/SP, Unicamp, USP e FGV/SP, 2002.

\section{Figura 3}

Dissertações e teses sobre saúde por temáticas na FGV/SP, PU C/SP, UNICAM P e USP (1990-2001).

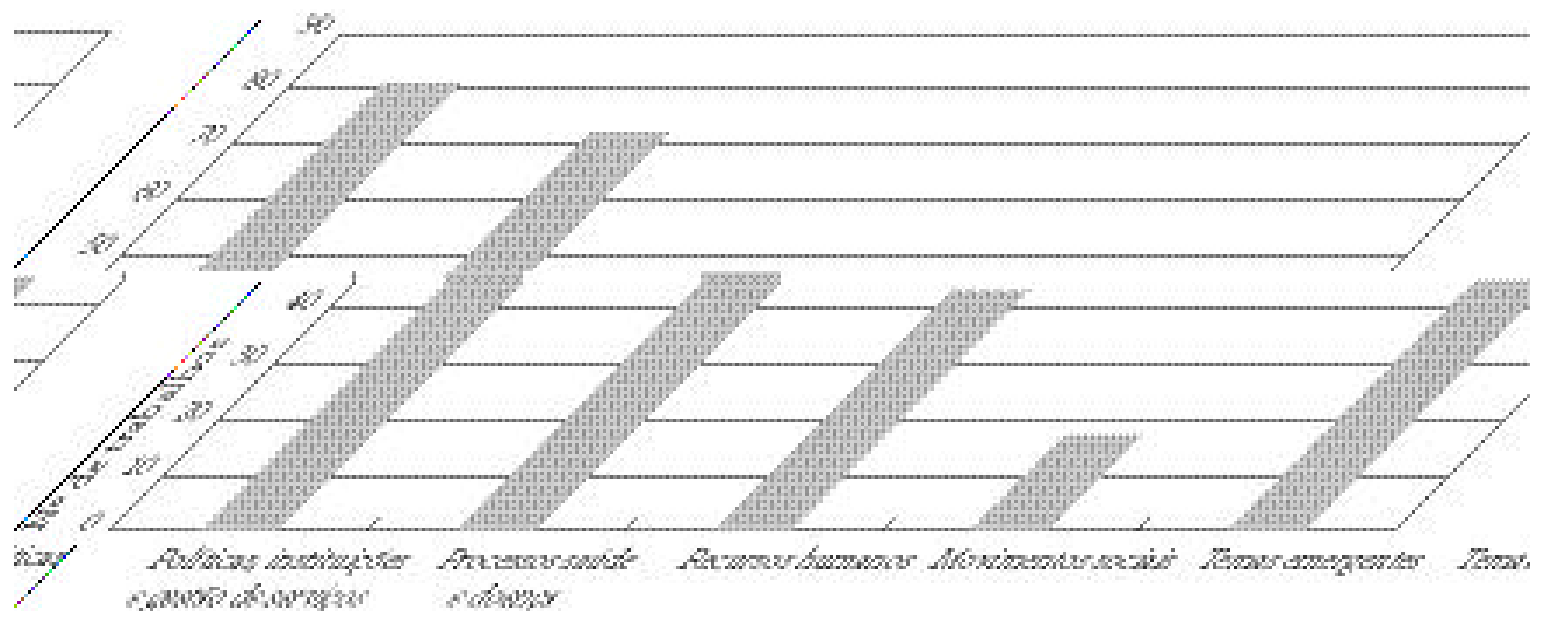

Fonte: Bibliotecas PUC/SP, Unicamp, USP e FGV/SP, 2002 
Entre os "temas emergentes", os mais freqüentemente abordados pela produção dos pós-graduandos das quatro instituições foram as questões relativas à saúde e trabal ho e à qualidade de vida e ambiente.

Durante a década de 1990 houve um interesse variado pelos cinco grupos de temas: a) os temas relativos às "políticas, instituições e gestão de serviços" e "formação de recursos humanos" dobraram na segunda metade da década; b) os temas referentes aos "processos saúde/ doença" tiveram praticamente o mesmo interesse dos pós-graduandos das ciências humanas e sociais, na primeira e na segunda metade dos anos $90 ; c) 0$ interesse pel os temas referentes aos "movimentos sociais" foi pequeno durante toda a década; d) o interesse pel os "temas emergentes" foi incrementado na segunda metade da década de 1990 e continuou nos anos de 2000 e 2001.

Quando comparamos a produção da pósgraduação das ciências sociais em saúde na área de saúde coletiva, (M inayo, 2000), com a produção da pós-graduação no interior das ciências sociais e humanas, podemos perceber que tanto no interior da área de saúde coletiva, quanto no interior das ciências sociais e humanas, os temas mais abordados referiram-se às "políticas, instituições e gestão de serviços", seguidos dos temas referentes aos "processos saúde/doença". Os temas referentes à "formação de recursos humanos" foram os terceiros mais abordados nas duas áreas, mas, proporcionalmente, foram mais abordados no interior das ciências sociais e humanas, em virtude do grande interesse dos pós-graduandos da área de administração, em especial da FGV/SP, por essa temática.

Os "temas emergentes" apareceram em quarto lugar nas áreas de ciências sociais e humanas e, dentre estes, principalmente os referentes às relações entre saúde e trabalho e qualidade de vida e ambiente. Poucos trabal hos referiram-se às questões da ética ou da violência no interior das ciências sociais e humanas, ao contrário do que foi assinalado por M inayo, (2000), quando analisou a produção acadêmica das ciências sociais na saúde coletiva.

\section{Experiências e reflexões dos orientadores de ciências sociais e humanas sobre as abordagens das temáticas de saúde}

\section{Saúde como objeto das ciências sociais e humanas}

Reconhecem as professoras $M$ ary Jane Spink e M aria H elena Villas-Boas Concone (PUC/SP) que a saúde é um tema antigo nas áreas de psicologia e antropologia, eno caso da antropologia, isto pode ser comprovado na literatura de origem americana ou inglesa dos anos $50 . \mathrm{No}$ Brasil a antropologia e a psicologia interessaram-se, desde o começo do século 20, pelos temas referentes à saúde mental. N os estudos antropológicos sobre medicina popular é possível identificar mais trabalhos de pesquisadores interessados no folclore, e uma relação um pouco conflituosa com as pesquisas antropológicas. Nos anos 80 houve um crescimento do interesse da antropologia pela questão dos vários tipos de terapias e do valor social dos alimentos. No entanto, a antropologia no Brasil era vista com certa estranheza pel os médicos, ao contrário da antropologia médica americana, inserida nos hospitais.

Os primei ros contatos dos orientadores entrevistados com as questões da saúde aconteceram de maneira variada: al guns, por serem professores em universidades que têm cursos de medicina ou enfermagem, passaram a ministrar as disciplinas de antropologia ou sociologia que foram introduzidas nos cursos da área de saúde ao final dos anos 60 e início dos anos 70, como foi o caso da professora Villas-Boas Concone. Outros começaram o contato pela própria área de saúde pública, nos cursos de aprimoramento, especialização ou pós-graduação para profissionais de saúde, como ocorreu com a professora M ary Jane Spink ( $p$ sicologia social/PUC/ SP. Ou ainda, vinculando-se a pesquisas que estavam sendo desenvolvidas na área de saúde, como o caso da professora M aria H elena Oliva Augusto (sociologia/USP), em uma pesquisa de professores do D epartamento de M edicina Preventiva da Faculdade de M edicina da USP na área de saúde e trabalho.

Poucos tomaram contato com as temáticas da área de saúde na pós-graduação de suas respectivas áreas nas ciências sociais e humanas, com outros professores que já pesquisavam temas da saúde e incentivaram a formação de linhas de pesquisa, como foi o caso da professora 
Avelino (história/PUC/SP). 0 professor Barros de Castro (economia/U nicamp) começou sua vinculação com a saúde através do Núcleo de Pesquisa em Políticas Públicas (NEPP) coordenado pela professora Sônia Draibe. E outros, ao contrário, primeiro tiveram uma trajetória longa nos cursos da área de saúde diretamente, e só mais recentemente se vincularam institucionalmente às áreas de ciências humanas e sociais, como foi o caso da professora M alik (Escola de Administração de Empresas/FGV/SP) e do professor Prates Junqueira (pós-graduação em administração/PUC/SP).

As experiências desses professores como orientadores de temas de saúde começou de forma esporádica, em seus respectivos programas de pós-graduação, sem que fossem definidas linhas de pesquisa: no geral, os próprios candidatos ou outros professores dos programas de pós-graduação que os procuravam sabiam que esses professores entrevistados tinham experiência com questões da área de saúde, porque haviam ministrado cursos de graduação ou pós-graduação lato sensu na área de saúde, ou participado de pesquisas em instituições da área de saúde. De qualquer maneira, esses professores orientadores já eram conhecidos na área de saúde coletiva ou saúde pública e, de certa forma, se constituíram em referências nas áreas de ciências sociais e humanas para os candidatos formados em vários cursos de graduação da área de saúde. Em muitos programas de pósgraduação das áreas de ciências sociais e humanas, a maioria dos candidatos que quer pesquisar sobre temas de saúde são originários da própria área de saúde, no geral profissionais, raramente com perfil acadêmico ou docente. Em alguns dos programas pesquisados esse perfil permanece até hoje, mas em outros como o da psicologia social da PUC/SP, do Instituto de Economia da U nicamp e da Escola de Administração da FGV, PROAHSA, relatam os professores entrevistados que está crescendo 0 afluxo de candidatos originários de cursos de graduação das áreas de ciências humanas e sociais.

As razões para essas mudanças do perfil da demanda dos pós-graduandos podem ser atribuídas à tentativa que os orientadores estão desenvolvendo no momento, nos cursos de pósgraduação, ou às novas abordagens que introduziram, ou ainda a mudanças na própria área de saúde, em especial na gestão de serviços. Assim, a abordagem das questões referentes à saúde mental tem trazido alunos com formação básica em psicologia para a pós-graduação em psicologia social, a abordagem do setor saúde como um complexo econômico e a tradição da economia da Unicamp em dedicar-se à economia política e aos problemas macroeconômicos têm interessado al guns economistas e administradores de formação básica nessas áreas para a produção acadêmica sobre temas da área de saúde. A modernização do setor privado na saúde, a expansão dos planos e seguros de saúde, a preocupação com o marketing dos serviços, as novas relações entre o setor público e 0 privado na saúde, em especial as organizações sociais de interesse público, têm atraído alunos dos cursos de graduação da FGV/SP eadministração da PU C/SP, para a pós-graduação nas linhas de administração de serviços de saúde.

As dificuldades apresentadas pelos alunos com formação básica nas diferentes áreas da saúde quando passam a integrar os programas de pós-graduação das áreas de ciências sociais e humanas podem ser definidas como de três tipos: específicas, porque decorrem da não familiaridade com as teorias, os métodos e as leituras próprias das ciências humanas e sociais. Esses fatos foram mais apontados pelos professores de antropologia, história e administração, sendo necessário que os professores de pósgraduação ofereçam disciplinas básicas para suprir essas dificuldades. Um outro tipo de dificuldade, não específica dos candidatos da área de saúde, mas de todos os demais, é a falta de experiência em pesquisa. $E$ ainda um terceiro tipo de dificuldade foi apontado particularmente nos candidatos da área de saúde que procuram a pós-graduação em administração, que é de caráter mais político e ideológico: a resistência, não aceitação, ou pelo menos desconhecimento, da bibliografia de administração de empresas, em geral de origem americana.

A tualmente os temas de saúde mais trazidos pelos candidatos referem-se às políticas de saúde, saúde mental, questões de gênero e de saúde, redução de risco, saúde do trabalhador, financiamento e custos na saúde, organização dos serviços e gestão. Particularmente na antropologia ena psicologia social, a questão da Aids concentrou as atenções dos interessados na pós-graduação durante toda a década de 1990.

Os espaços para a produção sobre temas de saúde nos fóruns próprios das ciências sociais e humanas, como congressos e seminários, são muito pequenos e no geral quando os temas aparecem, o fazem de maneira dispersa nos vários Grupos de Trabalho, não chegando a constituir um grupo próprio. Isso pode ser percebi- 
do nos encontros da Anpocs e Anpad. Na Anpec, no entanto, há um grupo específico de economia da saúde, e na antropologia tem havido maior facilidade, porque a ABA (Associação Brasileira de Antropologia) tem grupos de trabalho para questões que são importantes também para a saúde, como a questão do corpo, terapias ea preocupação com a Aids. Lembramos ainda, que a Associação Brasileira de Antropologia M édica tem realizado seus encontros, com participação de pesquisadores da antropologia e da saúde. No caso da administração há um grupo de pesquisadores no Rio Grande do Sul que está procurando criar um Grupo de TrabaIho sobre as questões de saúde. Já nos encontros da Abrapso (Associação Brasileira de Psicologia Social), pelo fato de a psicologia estar inserida no campo da saúde, encontra-se um grande número de trabal hos sobre saúde, especialmente saúde mental.

Alguns orientadores analisam que a saúde tem sido considerada uma "questão menor" nas ciências sociais ou uma área de conflito e disputa de saberes e poderes, como éo caso da área de administração, o que acaba prejudicando a comunicação e a produção acadêmica conjunta e potencializada entre as duas áreas.

Importante assinalar também que os pesquisadores das áreas de ciências sociais e humanas se queixaram de que seus trabalhos não encontram espaço nos congressos de saúde coletiva, e em especial nos encontros de ciências sociais em saúde, o que os deixa muitas vezes isolados, sem interlocução na área de ciências sociais e humanas e também na área de saúde, muito embora reconheçam que há uma produção das ciências sociais em saúde bastante organizada e de fácil acesso na saúde coletiva.

Por outro lado há o reconhecimento, principalmente da antropologia, de que na área de saúde tem se produzido trabalhos de caráter teórico ou metodológico de excelente qualidade. Já na história, há a impressão de que os trabal hos ditos "históricos" na saúde e especialmente na temática de história da medicina, produzidos por médicos, são bastante descritivos e revelam desconhecimento dos métodos de história.

Algumas estratégias para interessar alunos de graduação das ciências sociais e humanas pelas questões da saúde têm dado resultados, pois incentivam os alunos a procurarem professores de pós-graduação com projetos de pesquisa de mestrado ou doutorado sobre saúde: são elas a criação de disciplinas optativas sobre saúde na graduação ou projetos de iniciação científica. Por outro lado, alguns orientadores percebem que às vezes os professores é que têm sido pressionados pelos al unos de graduação para a discussão de temas de saúde; mas no geral, são pessoas que trabalham profissionalmente na área de saúde, e que estão matriculados nos cursos de graduação em ciências sociais ehumanas.

Seminários temáticos na pós-graduação sobre ciências sociais em saúde, administração de serviços de saúde, economia da saúde, têm tido sucesso nas áreas de sociologia da U SP, Instituto de Economia da U nicamp ou da FGV/SP, como captadores de futuros pós-graduandos interessados nas questões de saúde, entre os graduandos de ciências sociais e humanas.

\section{Conclusão}

Durante os anos 90 houve uma razoável produção de dissertações de mestrado e teses de doutorado nas áreas de pós-graduação das ciências sociais e humanas sobre temáticas de saúde, na USP, Unicamp, PU C/SP e FGV/SP.

As temáticas mais abordadas nesses trabaIhos apresentam pequenas diferenças quando comparadas às das ciências sociais no interior da saúde coletiva, mas que devem ser assinaladas: proporcionalmente a produção sobre "políticas, instituições e gestão de serviços" foi menor nas ciências humanas e sociais, mas a produção sobre os "processos saúde/doença" e "movimentos sociais" foi um pouco maior. Chama a atenção que a "formação de recursos humanos" apareceu também mais nesses trabalhos, principalmente pela forte presença dessa temática nas dissertações e teses da área de administração de empresas. E quanto aos "temas emergentes", a preferência pelas questões que relacionam "saúde e trabalho" e "qualidade de vida e ambiente".

Os professores que orientaram esses trabaIhos tiveram contato com as questões de saúde na própria área de saúde pública, saúde coletiva ou nos departamentos de medicina preventiva, pouco antes de se inserirem nas instituições de ensino e pesquisa de ciências sociais e humanas, ou quando já eram professores dessas instituições. Alguns, no entanto, fizeram sua carreira na saúde pública/coletiva e, só posteriormente, passaram a ministrar cursos nas instituições de ensino e pesquisa em ciências sociais e humanas, em particular na área de administração. 
A maioria dos autores dessas dissertações de mestrado e teses de doutorado sobre temáticas de saúde fez seus cursos de graduação na área de saúde, e procurou a pós-graduação nos programas de ciências sociais e humanas. No entanto, mais recentemente, alguns pós-graduandos com formação de graduação em ciências sociais e humanas têm produzido suas teses e dissertações sobre temas de saúde.

0 interesse pelos temas de saúde em áreas como as de antropologia e psicologia social é antigo, ou alguns temas fazem parte da própria área, como é o caso da saúde mental para a psicologia social. Temas como a Aids, saúde mental, terapias e organização de serviços têm interessado aos cientistas sociais e das áreas de humanas: psicologia, história, administração, educação e serviço social.

No caso da administração, embora muitas vezes ainda haja desconhecimento ou até um certo conflito de saberes e poderes na abordagem de temáticas de saúde, a questão da ampliação do setor privado na saúde tem interessado os administradores a se voltarem para a administração dos serviços de saúde. $\mathrm{Na}$ área de economia, a abordagem do setor saúde como um complexo econômico tem potencial para interessar os economistas pelo tema nas próximas décadas.

Apesar de se constatar uma razoável produção acadêmica sobre a saúde nos programas de pós-graduação em várias áreas das ciências so- ciais e humanas, nos fóruns próprios dessas áreas, isto é, nas associações, congressos e encontros, ainda há pouco espaço para a apresentação dessa produção.

A criação de disciplinas optativas nos cursos de pós-graduação ou de graduação em ciências sociais e humanas tem demonstrado potencial para interessar graduandos dessas áreas pelas temáticas de saúde, o mesmo ocorrendo com a estratégia de vincular alunos de graduação a pesquisas sobre saúde, na modalidade de iniciação científica. M as ainda a maior parte dos cientistas sociais e das áreas de humanas que se inserem na área de saúde tem feito sua formação de pós-graduação na saúde pública e saúde coletiva.

A ampliação do âmbito da Comissão deCiências Sociais da Abrasco para abranger também os trabalhos e pesquisadores das ciências humanas e a definição de temas transversais a várias dessas áreas para o II Encontro de Ciências Sociais em Saúde realizado na Fundação Oswaldo Cruz em dezembro de 2002 no Rio de Janeiro, revela-se como uma decisão acertada, diante da amplitude da produção das pós-graduações analisadas.

Este artigo apresentou resultados do levantamento realizado em apenas quatro instituições de ensino e pesquisa de São Paulo, PUC/ SP, USP, U nicamp e FGV/SP. Esperamos que possam contribuir para o conhecimento desse tipo de produção nas outras instituições de ensino e universidades do país. 


\section{Referências bibliográficas}

Canesqui AM (org.) 1995. Dilemas e desafios das ciências sociais na saúde coletiva. Ed. Hucitec-Abrasco, São Paulo-Rio de Janeiro, 165pp.

Canesqui AM (org.) 1997. Ciências sociais e saúde. Ed. Hucitec-Abrasco, São Paulo-Rio de Janeiro, 287pp.

$\mathrm{H}$ all $\mathrm{O}$ 1948. The stages of medical career.The American Journal of Sociology 53:327-336.

Luz M T 2000. A produção científica em ciências sociais e saúde: notas preliminares. Saúde em D ebate. Revista do Centro Brasileiro de Estudos de Saúde 24(55):5468, maio/ago.

M arsiglia RM G \& Spinelli SP 1995. As ciências sociais em saúde e o ensino, pp. 123-132. In AM Canesqui, (org.). Dilemas e desafios das ciências sociais na saúde coletiva. Ed. Hucitec-Abrasco, São Paulo-Rio de Janeiro, 165pp.

M inayo M CS 2000. Ciências sociais em saúde no Brasil: vida longa e recente relevância. Saúde em D ebate. Revista do Centro Brasileiro de Estudos de Saúde 24(55): 24-53, maio/ago.
Molina G 1974. Historia y sociologia de la medicina de $H$ enry E. Sigerist (seleções). Tradução, seleção e edição dos textos por M olina. Bogotá.

Nunes ED 1999. Sobre a sociologia da saúde. Ed. Hucitec, São Paulo, 234pp.

Rosen G 1958. U ma história da saúde pública. (Tradução do original inglês de 1958). Ed. Hucitec-Unesp-Abrasco, São Paulo-Rio de Janeiro.

Rosen G 1980. Da polícia médica à medicina social. Ed. Graal, Rio de Janeiro.

Sigerist HE 1929. The special position of the sick, pp. 922. In MI Roemer (org.). Henry Sigerist on the sociology of M edicine. MD. Publications, N ova York.

Sigerist HE 1946. The place of the physician in modern society, pp. 65-74. In MI Roemer (org.). Op. cit.

Stern BJ 1940. Society and medical progress. Columbia University Press, N ova York.

Artigo apresentado em 2/7/2002

Aprovado em 8/9/2002

Versão final apresentada em 20/9/2002 